\title{
PTEN activity could be a predictive marker of trastuzumab efficacy in the treatment of ErbB2-overexpressing breast cancer
}

\author{
T Fujita*,', H Doihara', K Kawasaki', D Takabatake', H Takahashi', K Washio', K Tsukuda', Y Ogasawara' and \\ N Shimizu' \\ 'Department of Cancer and Thoracic Surgery, Okayama University Graduate School of Medicine and Dentistry, 2-5-I Shikata-cho, 700-8558 Okayama, \\ Japan
}

Trastuzumab is the only HER2/neu-directed therapy to have received Food and Drug Administration approval for the treatment of patients with metastatic breast cancer. The efficacy of trastuzumab depends on the HER2/neu status of the tumour and the patient's prior treatment, but even when patients are selected on the basis of HER2/neu gene amplification, the single-agent response rate ranges from 12 to $30 \%$ and few patients respond to trastuzumab monotherapy. Here, we propose PTEN as a predictive biomarker for trastuzumab efficacy. Human breast cancer SKBR3 and drug-resistant SKBR3/R cells were investigated. We also examined clinical samples from patients who had been treated with trastuzumab and analysed the relationship between trastuzumab efficacy and PTEN level. The PI3KJAkt signalling pathway was observed to be highly active in the drug-resistant cells, and their level of PTEN was low. Delivery of antisense PTEN duplex siRNA significantly decreased the trastuzumab chemosensitivity of parental SKBR3 cells, and marked activation of Akt signalling pathway was also recognised. Moreover, immunohistochemical investigation revealed that trastuzumab treatment was remarkably successful in cells with elevated PTEN expression. Along with the immune-system-associated cytotoxic mechanism, several mechanisms have been proposed for the effect of trastuzumab. PTEN activity might play an important and major role in its HER2/PI3K/Akt-mediated antitumour effect, and could be a useful biomarker for predicting the efficacy of trastuzumab in the treatment of breast cancer.

British Journal of Cancer (2006) 94, 247-252. doi:I0.1038/sj.bjc.6602926 www.bjcancer.com

Published online 10 January 2006

(c) 2006 Cancer Research UK

Keywords: breast cancer; erbB2; trastuzumab; PTEN; Akt

PTEN (phosphatase and tensin homologue deleted on chromosome 10, also known as MMAC and TEP1) is a recently discovered tumour-suppressor gene located on chromosome 10q23.3. PTEN mutations have been implicated in variety of human cancers including endometrial cancer (30-50\%) (Risinger et al, 1997; Tashiro et al, 1997), high-grade glioma (60-80\%) (Louis and Gusella, 1995) and prostate cancer (29\%) (Cairns et al, 1997), and homozygous deletion of the PTEN gene causes embryonic lethality (Podsypanina et al, 1999). PTEN also antagonises phosphatidylinositol 3 kinase (PI3K) function and negatively regulates Akt activity. Restoration of PTEN expression in PTEN-null cells inhibits Akt activity and tumour formation (Li and Sun, 1998; Lu et al, 1999).

The HER2/neu oncogene, the second member of the epidermal growth factor receptor family, encodes a transmembrane tyrosinekinase receptor. Overexpression of HER2/neu, which is seen in approximately $30 \%$ of breast cancers, is associated with poor overall survival ( $\mathrm{Yu}$ and Hung, 2000) and in particular with increased metastatic potential and resistance to chemotherapeutic agents. Several reports have described the significance of PI3K and the Akt pathway in HER2/neu signalling. PI3K and Akt have been

*Correspondence: Dr T Fujita; E-mail: cqs03255@nifty.com Revised 24 November 2005; accepted 29 November 2005; published online 10 January 2006 shown to play important roles in proliferation and cell survival and induce the expression of many cytokines (Downward, 1998).

Recent studies have demonstrated that resistance to trastuzumab treatment depends on the level of PTEN present (Crowder et al, 2004; Nagata et al, 2004; Pandolfi, 2004), with Nagata et al (2004) demonstrating that PTEN deficiency confers trastuzumab resistance in HER2/neu-overexpressing breast cancer cells.

Here we present the use of PTEN for predicting the efficacy of trastuzumab in drug-resistant and parental HER2/neu-overexpressing breast cancer cells. We also investigate the expression of PTEN in a clinical setting and discuss its role.

\section{MATERIALS AND METHODS}

\section{Cell culture and reagents}

Human breast cancer SKBR3 cells were obtained from the American Type Culture Collection (Manassas, VA, USA) and maintained in Dulbecco's modified Eagle's medium supplemented with fetal bovine serum $\left(10 \% \mathrm{vv}^{-1}\right)$, penicillin $\left(100 \mathrm{IU} \mathrm{ml}^{-1}\right)$ and streptomycin $\left(100 \mu \mathrm{g} / \mathrm{ml}^{-1}\right)$ incubated in $5 \% \mathrm{CO}_{2}$. Trastuzumab resistance was developed by continuous exposure to trastuzumab $\left(4 \mu \mathrm{g} \mathrm{ml}^{-1}\right.$ for SKBR3/4R and $8 \mu \mathrm{g} \mathrm{ml}^{-1}$ for SKBR3/8R) for 4 months, during which time the medium was replaced every 5 days 
and cells were passaged when $50 \%$ confluence was reached. Trastuzumab resistance was confirmed by dose-response studies as described below.

Trastuzumab was purchased from Chugai Seiyaku (Tokyo, Japan).

\section{Western blot analysis}

Cells $\left(5-10 \times 10^{6}\right)$ were washed twice with phosphate-buffered saline (PBS), then lysed with a hypotonic HEPES buffer $(250 \mu \mathrm{l}$, $10 \mathrm{~mm}, \mathrm{pH}$ 7.6) containing $\mathrm{KCl}(50 \mathrm{~mm})$, phenylmethane sulphonyl fluoride $(0.1 \mathrm{~mm})$, aprotinin $\left(0.5 \mu \mathrm{lml}^{-1}\right)$ and sodium orthovanadate $(0.1 \mathrm{~mm})$. After centrifugation, supernatants were adjusted to contain equal concentrations of protein, diluted with one volume of $5 \times$ SDS sample buffer and heated for $5 \mathrm{~min}$ at $95^{\circ} \mathrm{C}$. Samples (30 $\mu \mathrm{g}$ protein) were run on a $4-20 \%$ SDS-PAGE gel and electroblotted onto polyvinylidene fluoride membrane. Blots were blocked overnight in blocking solution (5\% nonfat milk powder, $0.1 \%$ Tween 20 in PBS) at $4{ }^{\circ} \mathrm{C}$. Afterwards, the blots were exposed to antibodies against HER2, PI3K, Akt, PTEN (Santa Cruz, CA, USA), p-PI3K or p-Akt (Cell Signalling) at 1000 -fold dilution in blocking solution ( $1 \mathrm{~h}$, room temperature). After extensive washing with blocking solution, blots were exposed to the appropriate secondary antibody at 10000 -fold dilution in blocking solution. After extensive washing, blots were examined using an enhanced chemiluminescence detection method (ECL kit, Amersham Pharmacia Biotech, Chandler, AZ, USA).

\section{Determination of cytotoxicity by MTT (3-[4,5-dimethylthyazol-2-yl]-2,5-diphenyl tetrazolium bromide) assay}

Cells were seeded at a concentration of $3 \times 10^{3}$ cells per well in flatbottomed 96-well microplates. After $24 \mathrm{~h}$, the cells were cultivated with trastuzumab for $120 \mathrm{~h}$ or paclitaxel for $12 \mathrm{~h}$, or left untreated, then their viability was determined using Promega's CellTiter $96^{\mathrm{R}}$ Aqueous One Solution Cell Proliferation Assay (Promega Corp., Madison, WI, USA).

\section{Delivery of PTEN duplex siRNA in vitro}

Duplex siRNA against PTEN (AF143314 4688 E06: 5'-AUGCCAA CAACAAGCUUCUUACAAUGCC- $3^{\prime}$ ) and control duplex siRNA (AF143314 4688 E07: 5'-AUGUACCAACCGAAUCUUACAUGCC-3') (Life Technologies, Rockville, MD, USA) were delivered in drugsensitive parental SKBR3 cells. Cells were plated in $100 \mathrm{~mm}$ dishes at $30 \%$ confluence and transfected with duplex siRNA (25 nM) using Oligofectamine (Life Technologies, Rockville, MD, USA) $48 \mathrm{~h}$ postplating. Cells were replated for individual assay $96 \mathrm{~h}$ postplating. PTEN expression was determined $120 \mathrm{~h}$ postplating and growth inhibition assay was performed after $72 \mathrm{~h}$ postplating followed by incubation with chemotherapeutic agents.

\section{Evaluation of HER2/neu status}

HER2/neu status was determined based on gene amplification and/ or immunohistochemical evaluation. HER2/neu gene amplification of the patients' samples was determined by fluorescence in situ hybridisation (FISH) using the PathVysion FISH assay (Vysis, IL, USA). Immunohistochemically, HER2/neu status was determined by Herceptest (DAKO, Tokyo, Japan).

\section{Immunohistochemical investigation of PTEN}

Clinical samples were used for immunohistochemical investigation with PTEN. Each sample was obtained from a surgical specimen of a patient who had received trastuzumab treatment in combination with paclitaxel in our department between 2001 and October 2005. For immunohistochemistry, paraffin sections were stained after microwave treatment by an intact method. Antibody against PTEN (Santa Cruz, CA, USA) was exposed overnight at $4{ }^{\circ} \mathrm{C}$ followed by treatment with the LSAB2 kit (DAKO Carpinteria, CA, USA) according to the manufacturer's instructions. The PTEN expression level was scored semiquantitatively based on staining intensity and distribution using the immunoreactive score (IRS) as described elsewhere (Friedrichs et al, 1993; Chui et al, 1996); briefly, IRS $=$ SI (staining intensity) $\times$ PP (percentage of positive cells). Staining intensity is assigned as 0 , negative; 1 , weak; 2 , moderate; 3 , strong. Percentage of positive cells is defined as 0 , $<1 \% ; 1,1-10 \% ; 2,11-50 \% ; 3,51-80 \%$; and $4,>80 \%$ positive cells. In all, 10 visual fields from different areas of each tumor were used for the IRS evaluation. Negative control slides without primary antibody were included for each staining. Normal breast epithelium or vascular endothelium known to express normal PTEN was used as positive control. Based on the IRS score, staining intensity was graded - (IRS 0-3), $1+$ (IRS 4-6), $2+$ (IRS 7-9) or 3+ (IRS 10-12).

\section{Statistical analysis}

Levels of statistical significance were evaluated using data from at least three independent experiments by using the two-tailed Student's $t$ test, Fisher's test and ANOVA. $P<0.05$ was considered to be statistically significant. All data were analysed using StatView for Windows (SAS Institute Inc., Cary, NC, USA).

\section{RESULTS}

\section{Growth inhibition of trastuzumab and paclitaxel in} parental and drug-resistant cells

The HER2/neu-overexpressing SKBR3 cells were treated with serial dilutions of trastuzumab and paclitaxel. The cells' survival at various drug concentrations are shown in Figure 1. In the drugresistant cells, mean cell viability was $79.6 \%$ (SKBR3/4R) and $128.6 \%$ (SKBR3/8R) at $2 \mu \mathrm{g} \mathrm{ml}^{-1}$ trastuzumab, which is markedly higher than the wild-type (SKBR3/WT) parental cells. Mean cell viabilities $\left(\mathrm{IC}_{50}\right)$ in parental and resistant SKBR3/4R cells were 8.25 and $23.79 \mu \mathrm{M}$ trastuzumab, respectively; the difference was statistically significant (Figure 2). However, the response of drug-resistant and parental cells to paclitaxel treatment did not differ significantly.

\section{Western blotting analysis of HER2/neu expression and downstream signal proteins}

Western blot was performed to detect expression of the downstream cascade pathway of HER2/neu2 in both resistant and parental cells. Expression of HER2/neu, PI3K, phosphorylated PI3K and nonphosphorylated Akt were the same in both cell types (Figure 3a). However, expression of phosphorylated Akt was significantly higher in the drug-resistant cell lines. Conversely, PTEN expression was decreased in the drug-resistant cells and this downregulation was more pronounced in the more resistant cells. Owing to the downregulation of PTEN, expression of phosphorylated Akt was increased and signal transduction of the Aktmediated pathway was activated (Figure $3 \mathrm{~b}$ ).

\section{PTEN deficiency and trastuzumab sensitivity in parental cells}

To elucidate the effect of PTEN on trastuzumab's efficacy, SKBR3/WT, 4R and 8R cells were transfected with PTEN antisense oligonucleotides, which effectively reduced endogenous 

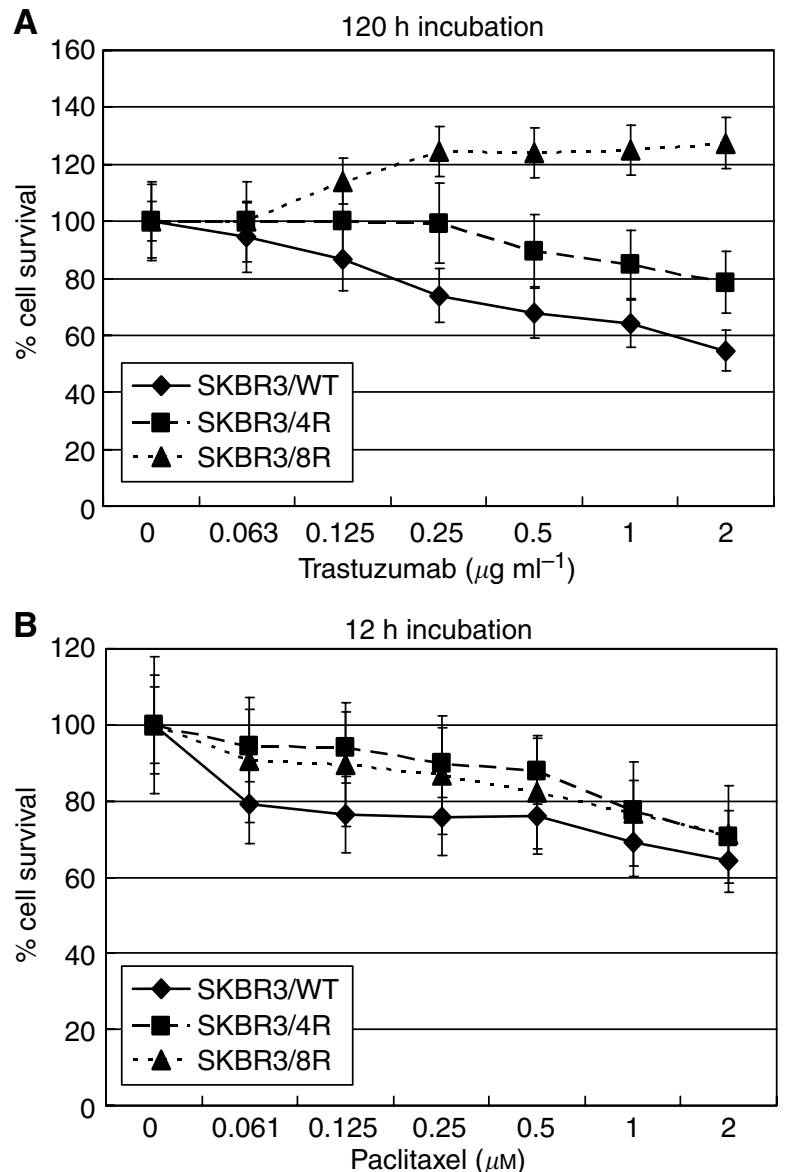

Figure I (A) Growth inhibition assays revealed that chemosensitivity to trastuzumab was decreased in drug-resistant SKBR3/4R and /8R cells ( $120 \mathrm{~h}$ incubation with trastuzumab after $24 \mathrm{~h}$ incubation with medium). (B) Chemosensitivity of paclitaxel was not significantly different in drug-resistant and parental SKBR3 cells $(12 \mathrm{~h}$ incubation with paclitaxel after $24 \mathrm{~h}$ incubation with medium).



Figure 2 The $I C_{50}$ value of trastuzumab in drug-resistant SKBR3/4R cells was statistically significantly higher than in parental cells.

PTEN expression compared with the cells transfected with control mismatched (MIS) oligonucleotides (Figure 4). To investigate whether PTEN activation contributes to trastuzumab's antiproliferation function, we compared cell growth between MIS control and PTEN antisense-delivered SKBR/WT cells
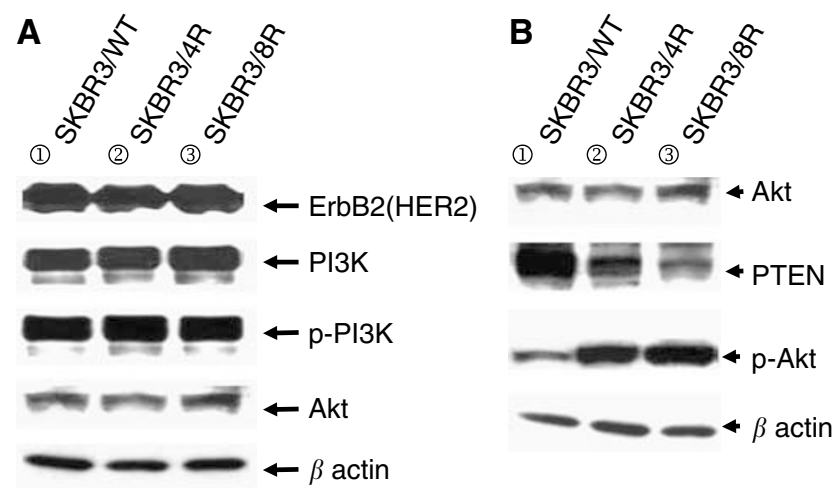

Figure 3 (A) Western blotting analysis revealed that the level of expression of the ErbB2/PI3K/Akt signal transduction pathway is the same in trastuzumab-resistant and parental cells. (B) Phosphorylated Akt activity was increased in drug-resistant SKBR3/R cells; PTEN expression was decreased in these cells.

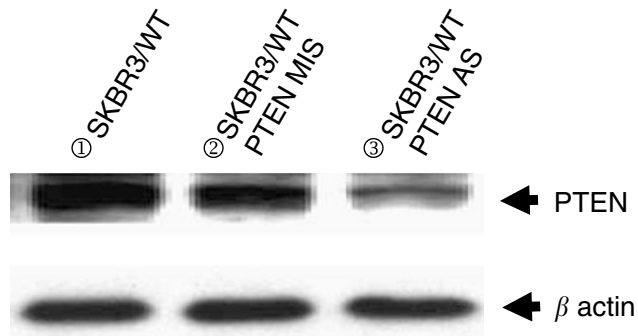

Figure 4 Western blotting analysis of antisense-delivered SKBR3/WT cells showed that expression of PTEN was inhibited by duplex antisensesiRNA delivery.

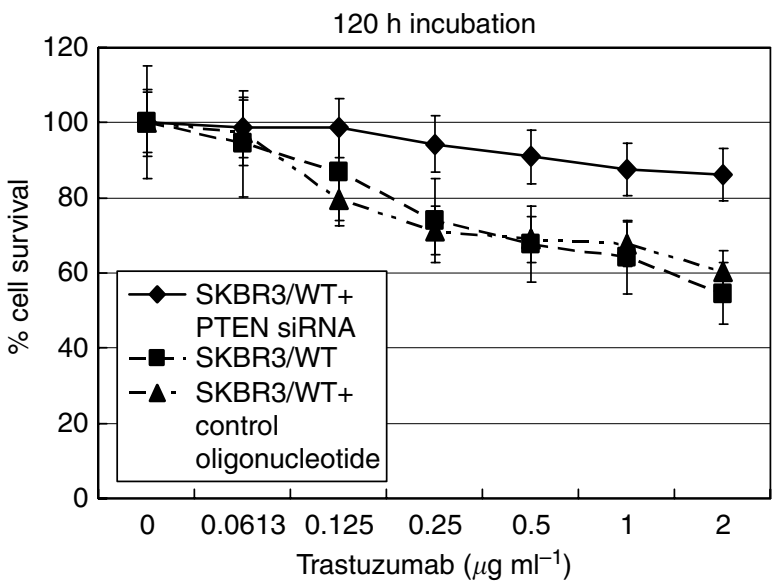

Figure 5 The growth inhibition curve of duplex antisense-siRNAdelivered SKBR3/NT cells shows decreased trastuzumab sensitivity (I $20 \mathrm{~h}$ incubation of trastuzumab $72 \mathrm{~h}$ postplating)

after trastuzumab treatment. PTEN antisense-delivered SKBR3/WT cells, which had reduced PTEN expression, showed significantly less growth inhibition with trastuzumab than MIS control-delivered cells with a normal level of PTEN expression (Figure 5). 
Western blotting analysis of HER2/neu expression and downstream signal proteins in PTEN-deficient cells

Western blot was used to detect the expression of the downstream cascade pathway of HER2/neu in PTEN antisense-delivered cells. Expression of nonphosphorylated Akt was the same in all cells, but expression of phosphorylated Akt was significantly higher in antisense-delivered SKBR3/WT cells than in drug-resistant cells (Figure 6).

\section{Growth inhibition effect of paclitaxel in PTEN-deficient cells}

The growth-inhibition effect of paclitaxel was analysed in PTENdeficient cells. No significant difference was observed between PTEN antisense-delivered cells and MIS control-delivered cells (Figure 7).

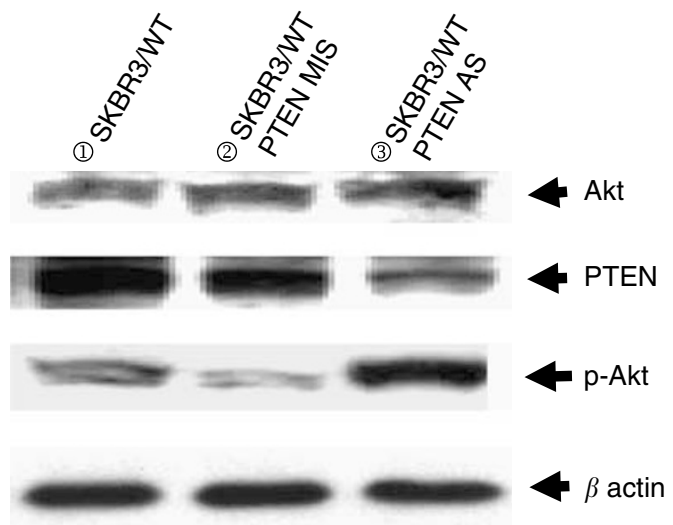

Figure 6 Western blotting analysis of antisense-delivered SKBR3/WT cells showed that expression of phosphorylated Akt was increased. This suggests that Akt activity is partly due to the level of PTEN in ErbB2overexpressing SKBR3 cells.

\section{Immunohistochemical analysis of PTEN in clinical specimens}

To explore the clinical significance of PTEN status in predicting response to trastuzumab-based therapy, we evaluated PTEN expression in $17 \mathrm{HER} 2 /$ neu-overexpressing primary breast carcinomas from patients who subsequently developed metastatic breast cancer and received trastuzumab plus paclitaxel therapy after preliminary evaluation, and in 20 other randomly selected breast cancer patients. Immunohistochemical staining revealed that PTEN expression in these tumours was heterogeneous. Thus, PTEN expression levels were semiquantified using IRS, calculated by multiplying the percentage of PTEN-positive cells (scored $0-4$ ) by the PTEN staining intensity (scored 1-3) (Chui et al, 1996). Immunoreactive scores range from 0 to 12 , representing the range of PTEN staining from an undetectable level in PTEN-deficient tumours to full expression in normal individuals (Figure 8). We

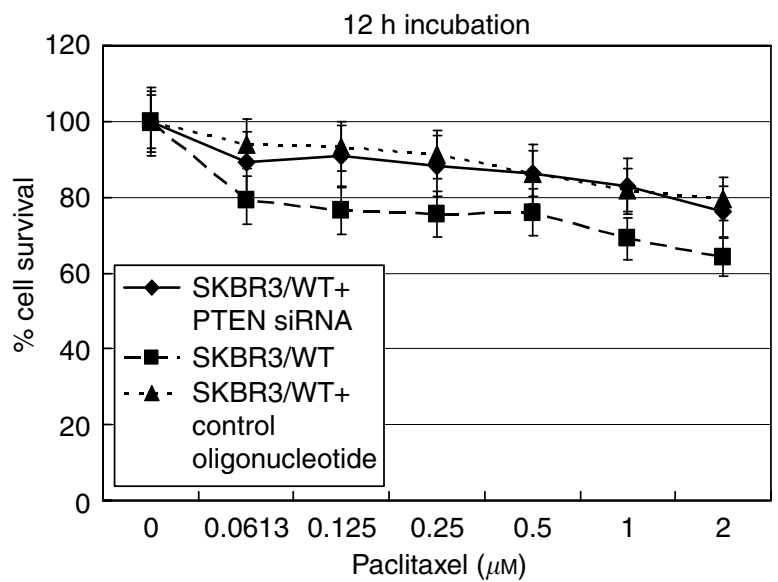

Figure 7 The growth inhibition rate of paclitaxel in antisense-delivered SKBR3/WT cells. No significant difference was observed for the growth inhibition effect of paclitaxel between antisense- and control oligonucleotide-delivered cells ( $12 \mathrm{~h}$ incubation of paclitaxel $72 \mathrm{~h}$ postplating).
$(-)$

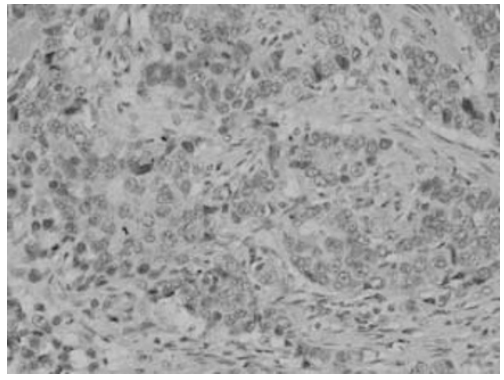

$(2+)$

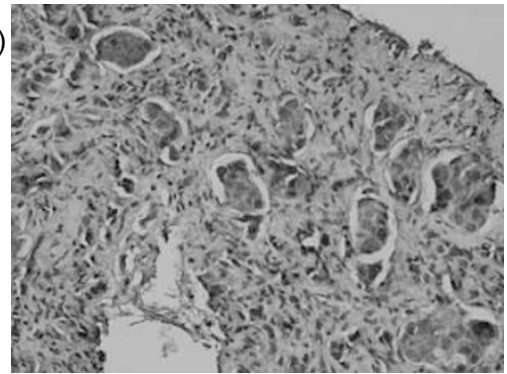

$(1+)$

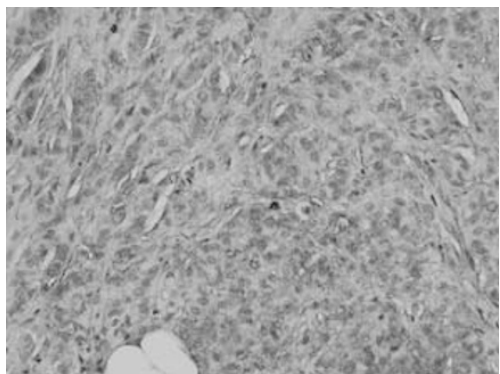

$(3+)$

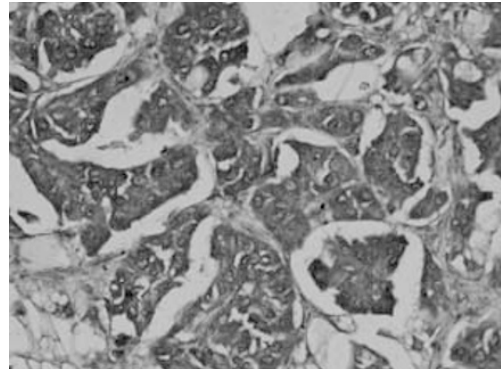

Figure 8 PTEN expression pattern in human breast tumours. ErbB2-overexpressing primary breast carcinomas from 17 patients who subsequently received trastuzumab plus pacliataxel were analysed. PTEN expression of these tumours was examined by IHC and semiquantified using immunoreactive scores. Representative tumour PTEN stainings (IRS $3+, 2+, 1+$, and - ). 


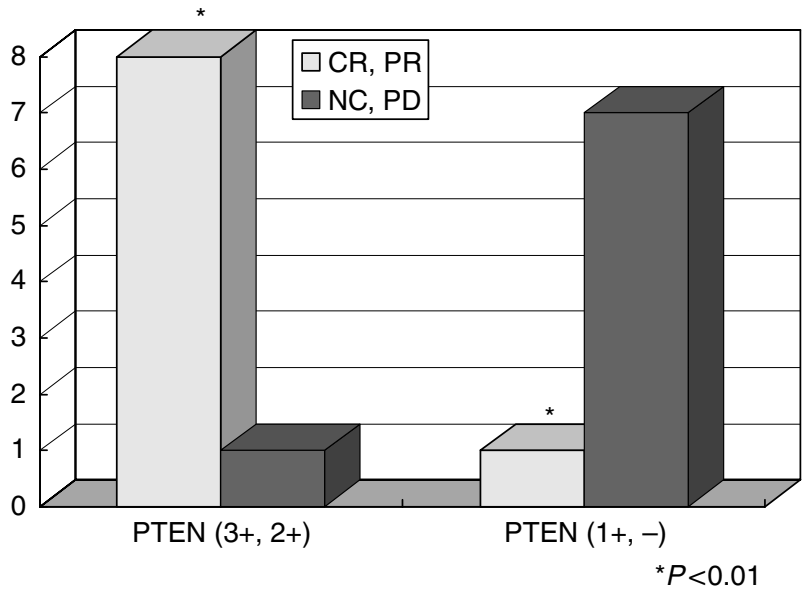

Figure 9 Immunohistochemical investigation of PTEN expression from patients with erbB2-overexpressing breast cancer treated with trastuzumab. We analysed 17 breast cancer tissue samples by immunohisto chemistry. In those cases with increased PTEN expression $(3+, 2+)$, the efficacy of trastuzumab-containing chemotherapy was remarkable and the response rate statistically significant (statistical analysis was performed with Fisher's test).

observed weak PTEN expression ( - and $1+$ : IRS $<7$ ) in $40.0-$ $49.1 \%$ of the tumours examined (eight out of 17 of the patients who received trastuzumab and paclitaxel, and eight out of 20 from the preliminary study), which is consistent with previous reports that $\sim 50 \%$ of breast cancers are PTEN deficient (Perren et al, 1999; Depowski et al, 2001). Of the 17 patients who received trastuzumab plus paclitaxel, those who had PTEN-deficient tumours $(-$ and $1+:$ IRS $<7)$ had significantly lower complete and partial response rates to trastuzumab therapy than those with PTEN-positive tumors $(2+$ and $3+$ or IRS $\geqslant 7 ; 12.50$ vs $88.89 \%$, $P=0.00337$ ) (Figure 9).

\section{DISCUSSION}

Several studies using either primary tumour tissue or established tumour cell lines have demonstrated a high frequency of PTEN mutation/deletion in various human cancers including brain, bladder, breast, prostate and endometrial cancers (Louis and Gusella, 1995; Cairns et al, 1997; Li et al, 1997; Risinger et al, 1997; Tashiro et al, 1997; Teng et al, 1997; Podsypanina et al, 1999; Mutter et al, 2000). PTEN has been found to be one of the most common targets of mutation in human cancer, with a mutation frequency approaching that of p53 (Bose et al, 1998). Human breast cancer is associated with a loss of heterozygosity or mutation of the PTEN gene, and decreased PTEN expression is associated with invasive breast cancer and poor prognosis (Singh et al, 1998; Garcia et al, 1999). The principal activity of PTEN is to dephosphorylate phosphatidylinositol 3,4,5-triphosphate (PIP3), produced by PI3K and a major activator of the cell survival kinase Akt. Thus, negative regulation of the PI3K pathway by PTEN is critical, and loss of PTEN function creates an environment conducive to tumorigenesis (Feilotter et al, 1999; Perren et al, 1999; Torres and Pulido, 2001). Currently, patients with metastatic breast cancer are selected for trastuzumab-based therapy if the primary tumour overexpresses the HER2/neu protein, or if FISH provides evidence of HER2/neu gene amplification (Di Cristofano et al, 1998). While trastuzumab therapy is not a cure for disseminated disease in HER2/neu amplification cases, major tumour regressions are often seen, particularly when the trastuzumab is given in combination with other chemotherapeutic agents. However, in spite of patient selection on the basis of HER2/ neu FISH or protein overexpression, less than $30 \%$ of patients respond to trastuzumab monotherapy. Taking these observations into account, HER2/neu gene amplification is a necessary biomarker but not sufficient to predict the efficacy of trastuzumab. It is probable that trastuzumab efficacy is also controlled by other biomarkers associated with the downstream pathway of HER2/neu. Some recent reports have demonstrated that PTEN activation contributes to tumour inhibition by trastuzumab, and loss of PTEN predicts trastuzumab resistance in vitro, in vivo and in the clinical setting (Crowder et al, 2004; Nagata et al, 2004; Pandolfi, 2004). According to Nagata et al (2004), activation of PTEN in HER2/neu-amplified breast cancer could contribute to the efficacy of trastuzumab; it was also demonstrated that efficacy of trastuzumab in clinical samples of HER2/neu-amplified breast cancer is significantly associated with PTEN expression.

In this in vitro study, we investigated the relationship between trastuzumab's efficacy and PTEN status with a drug-resistant cell model established by continuous exposure to trastuzumab. Our method for the establishment of trastuzumab-resistant HER2/neuoverexpressing breast cancer cells is similar to that of Nahta et al (2004) and could provide a model for the investigation of the molecular mechanisms of trastuzumab resistance. As in previous reports (Crowder et al, 2004; Nagata et al, 2004; Pandolfi, 2004), our study revealed a remarkable difference in PTEN activity between drug-sensitive and -resistant cells. Selective inhibition of PTEN, which is strongly associated with induction of Akt activity, resulted in markedly decreased trastuzumab sensitivity. Furthermore, in the clinical samples from patients with HER2/neuoverexpressing breast cancer treated with trastuzumab, PTEN expression was strongly associated with trastuzumab efficacy. Therefore, based on this study and previous reports, trastuzumab efficacy is partly dependent on the PTEN status and degree of Akt and associated signal transduction activities in HER2/neu-overexpressing breast cancer.

Although we have not described the studies here, we have also investigated the effects of selective inhibition of other signal transduction pathways and transcription factors, including the Ras/Raf/MAPK pathway, JNK-medicated pathway and bcl2 apoptotic proteins, in trastuzumab-sensitive and -resistant SKBR3 cells. Little effect on trastuzumab efficacy was observed.

Other reports have discussed other predictive markers of trastuzumab efficacy (Albanell and Baselga 2001; Lu et al, 2001, 2004; Menendez et al, 2005; Vogel et al, 2005), including insulinlike growth factor-1 receptor (IGF-1R), a widely expressed protein tyrosine kinase with an important role in the suppression of apoptosis and stimulation of proliferation. However, these studies have not yet been expanded to the clinical setting (Lu et al, 2001). Our findings are strongly supported by clinical data and previous reports (Crowder et al, 2004; Nagata et al, 2004; Pandolfi, 2004) that PTEN-deficient tumours did not respond to trastuzumab. Therefore, PTEN, which is strongly associated with the PI3K/Akt signal transduction pathway, could predict the efficacy of trastuzumab in patients with excessive HER2/neu expression. Owing to the small cohort used in our study, further large-scale clinical investigations, including both histopathological and genetic aspects, should be carried out.

Several new molecular-target chemotherapeutic agents have been developed in recent years. Of these, selective inhibitors of Akt and mTOR have been examined in preclinical or Phase 1 clinical trials (Carraway and Hidalgo, 2004; Meuillet et al, 2004). According to the results of our study, the PTEN and Akt downstream pathway is critical for the efficacy of trastuzumab and these new agents targeted to the HER2/neu-mediated transduction pathway might give hope in overcoming trastuzumab resistance.

\section{ACKNOWLEDGEMENTS}

We thank and greatly appreciate Dr Naruto Taira and Ms Mayumi Okada for their valuable advice. 


\section{REFERENCES}

Albanell J, Baselga J (2001) Unraveling resistance to trastuzumab (Herceptin): insulin-like growth factor-I receptor, a new suspect. J Natl Cancer Inst 93: $1830-1832$

Bose S, Wang SI, Terry MB, Hibshoosh H, Parsons R (1998) Allelic loss of chromosome 10q23 is associated with tumor progression in breast carcinomas. Oncogene 17: $123-127$

Cairns P, Okami K, Halachmi S, Halachmi N, Esteller M, Herman JG, Jen J, Isaacs WB, Bova GS, Sidransky D (1997) Frequent inactivation of PTEN/ MMAC1 in primary prostate cancer. Cancer Res 57: 4997-5000

Carraway H, Hidalgo M (2004) New targets for therapy in breast cancer: mammalian target of rapamycin (mTOR) antagonists. Breast Cancer Res 6: $219-224$

Chui X, Egami H, Yamashita J, Kurizaki T, Ohmachi H, Yamamoto S, Ogawa M (1996) Immunohistochemical expression of the c-kit protooncogene product in human malignant and non-malignant breast tissues. Br J Cancer 73: $1233-1236$

Crowder RJ, Lombardi DP, Ellis MJ (2004) Successful targeting of ErbB2 receptor - is PTEN the key? Cancer Cell 6: 103-104

Depowski PL, Rosenthal SI, Ross JS (2001) Loss of expression of the PTEN gene protein product is associated with poor outcome in breast cancer. Mod Pathol 14: 672-676

Di Cristofano A, Pesce B, Cordon-Cardo C, Pandolfi PP (1998) Pten is essential for embryonic development and tumour suppression. Nat Genet 19: $348-355$

Downward J (1998) Ras signaling and apoptosis. Curr Opin Genet Dev 8: 49-54

Feilotter HE, Coulon V, McVeigh JL, Boag AH, Dorion-Bonnet F, Duboue B, Latham WC, Eng C, Mulligan LM, Longy M (1999) Analysis of the 10q23 chromosomal region and the PTEN gene in human sporadic breast carcinoma. Br J Cancer 79: 718-723

Friedrichs K, Gluba S, Eidtmann H, Jonat W (1993) Overexpression of p53 and prognosis in breast cancer. Cancer 72: $3641-3647$

Garcia JM, Silva JM, Dominguez G, Gonzalez R, Navarro A, Carretero L, Provencio M, Espana P, Bonilla F (1999) Allelic loss of the PTEN region (10q23) in breast carcinomas of poor pathophenotype. Breast Cancer Res Treat 57: $237-243$

Li DM, Sun H (1998) PTEN/MMAC1/TEP1 suppresses the tumorigenicity and induces G1 cell cycle arrest in human glioblastoma cells. Proc Natl Acad Sci USA 95: 15406 - 15411

Li J, Yen C, Liaw D, Podsypanina K, Bose S, Wang SI, Puc J, Miliaresis C, Rodgers L, McCombie R, Bigner SH, Giovanella BC, Ittmann M (1997) PTEN, a putative protein tyrosine phosphatase gene mutated in human brain, breast, and prostate cancer. Science 275: $1943-1947$

Louis DN, Gusella JF (1995) A tiger behind many doors: multiple genetic pathways to malignant glioma. Trends Genet 11: $412-415$

Lu Y, Lin YZ, LaPushin R, Cuevas B, Fang X, Yu SX, Davies MA, Khan H, Furui T, Mao M, Zinner R, Hung MC, Steck P, Siminovitch K, Mills GB (1999) The PTEN/MMAC1/TEP tumor suppressor gene decreases cell growth and induces apoptosis and anoikis in breast cancer cells. Oncogene 18: $7034-7045$

Lu Y, Zi X, Pollak M (2004) Molecular mechanisms underlying IGF-Iinduced attenuation of the growth-inhibitory activity of trastuzumab (Herceptin) on SKBR3 breast cancer cells. Int J Cancer 108: 334-341
Lu Y, Zi X, Zhao Y, Mascarenhas D, Pollak M (2001) Insulin-like growth factor-1 receptor signaling and resistance to trastuzumab (Herceptin). J Natl Cancer Inst 93: $1852-1857$

Menendez JA, Vellon L, Lupu R (2005) Targeting fatty acid synthase-driven lipid rafts: a novel strategy to overcome trastuzumab resistance in breast cancer cells. Med Hypotheses 64: 997-1001

Meuillet EJ, Ihle N, Baker AF, Gard JM, Stamper C, Williams R, Coon A, Mahadevan D, George BL, Kirkpatrick L, Powis G (2004) In vivo molecular pharmacology and antitumor activity of the targeted Akt inhibitor PX-316. Oncol Res 14(10): 513-527

Mutter GL, Lin MC, Fitzgerald JT, Kum JB, Baak JP, Lees JA, Weng LP, Eng C (2000) Altered PTEN expression as a diagnostic marker for the earliest endometrial precancers. J Natl Cancer Inst 92: 924-930

Nagata Y, Lan KH, Zhou X, Tan M, Esteva FJ, Sahin AA, Klos KS, Li P, Monia BP, Nguyen N, Hortobagyi GN, Hung MC, Yu D (2004) PTEN activation contributes to tumor inhibition by trastuzumab, and loss of PTEN predicts trastuzumab resistance in patients. Cancer Cell 6 : $117-127$

Nahta R, Esteva FJ (2004) In vitro effects of trastuzumab and vinorelbine in trastuzumab-resistant breast cancer cells. Cancer Chemother Pharmacol 53: $186-190$

Pandolfi PP (2004) Breast cancer - loss of PTEN predicts resistance to treatment. N Engl J Med 351: $2337-2338$

Perren A, Weng LP, Boag AH, Ziebold U, Thakore K, Dahia PL, Komminoth P, Lees JA, Mulligan LM, Mutter GL, Eng C (1999) Immunohistochemical evidence of loss of PTEN expression in primary ductal adenocarcinomas of the breast. Am J Pathol 155: 1253-1260

Podsypanina K, Ellenson LH, Nemes A, Gu J, Tamura M, Yamada KM, Cordon-Cardo C, Catoretti G, Fisher PE, Parsons R (1999) Mutation of Pten/Mmac1 in mice causes neoplasia in multiple organ systems. Proc Natl Acad Sci USA 96: 1563-1568

Risinger J, Hayes AK, Berchuck A, Barrett JC (1997) PTEN/MMAC1 mutations in endometrial cancers. Cancer Res 57: 4736-4738

Singh B, Ittmann MM, Krolewski JJ (1998) Sporadic breast cancers exhibit loss of heterozygosity on chromosome segment 10q23 close to the Cowden disease locus. Genes Chromosomes Cancer 21: 166-171

Tashiro H, Blazes MS, Wu R, Cho KR, Bose S, Wang SI, Li J, Parsons R, Ellenson LH (1997) Mutations in PTEN are frequent in endometrial carcinoma but rare in other common gynecological malignancies. Cancer Res 57: $3935-3940$

Teng DH, Hu R, Lin H, Davis T, Iliev D, Frye C, Swedlund B, Hansen KL, Vinson VL, Gumpper KL, Ellis L, EL-Naggar A, Frazier M, Jasser S, Langford LA, Lee J, Mills GB, Pershouse MA, Pollack RE, Tornos C, Troncoso P, Yung WK, Fujii G, Berson A, Steck PA et al (1997) MMAC1/ PTEN mutations in primary tumor specimens and tumor cell lines. Cancer Res 57: 5221-5225

Torres J, Pulido R (2001) The tumor suppressor PTEN is phosphorylated by the protein kinase CK2 at its C terminus. J Biol Chem 276: $993-998$

Vogel CL, Reddy JC, Reyno LM (2005) Efficacy of trastuzumab. Cancer Res 65: 2044

Yu D, Hung MC (2000) Overexpression of ErbB2 in cancer and ErbB2targeting strategies. Oncogene 19: 6115-6121 